\title{
Rotational evolution of young pulsars due to superfluid decoupling
}

\author{
Wynn C. G. Ho ${ }^{\star}$ and Nils Andersson ${ }^{\star}$
}

\begin{abstract}
Pulsars are rotating neutron stars that are seen to slow down, and the spin-down rate is thought to be due to magnetic dipole radiation ${ }^{1,2}$. This leads to a prediction for the braking index $n$, which is a combination of the spin period and its first and second time derivatives. However, all observed values ${ }^{3}$ of $n$ are below the predicted value of 3 . Here we provide a simple model that can explain the rotational evolution of young pulsars, including the $n=2.51$ of the 958 -year-old pulsar in the Crab nebula ${ }^{4}$. The model is based on a decrease in the effective moment of inertia due to an increase in the fraction of the stellar core that becomes superfluid as the star cools through neutrino emission. The results suggest that future large radio monitoring campaigns of pulsars will yield measurements of the neutron star mass, nuclear equation of state and superfluid properties.
\end{abstract}

The core of a neutron star has densities near and above nuclear saturation and extends to $90 \%$ of the radius of the star; the remaining kilometre or so is the stellar crust. The core is composed of degenerate matter, mostly neutrons and a small fraction of protons and electrons (and possibly exotica, such as hyperons and deconfined quarks, which we do not consider here). Immediately after neutron-star formation, this matter is in a normal state owing to the high temperatures reached in stellar core collapse. However, neutron stars cool rapidly through the emission of neutrinos, and when the temperature drops below the (density-dependent) critical temperature for Cooper pairing, neutrons and protons form a superfluid and superconductor, respectively $y^{5,6}$. Superfluid neutrons rotate by forming quantized vortices, and the spatial distribution of these vortices determines the rotation rate of the superfluid core; for example, vortices migrate away from the stellar axis of rotation when the superfluid angular velocity $\Omega_{\text {sf }}$ decreases whereas $\Omega_{\mathrm{sf}}$ cannot change if the vortices are fixed in location, that is, when they are pinned. Meanwhile, normal matter (for example, in the crust) rotates at an angular velocity $\Omega$ that decreases as a result of energy loss from the stellar surface due to magnetic dipole radiation, that is, $\mathrm{d} E / \mathrm{d} t=-\beta \Omega^{4}$, where $\beta \approx B^{2} R^{6} / 6 c^{3}$ and $B$ and $R$ are the neutron star magnetic field and radius, respectively ${ }^{1,2}$.

A rapid decline in surface temperature was recently detected in the 330-year-old neutron star in the Cassiopeia A supernova remnant ${ }^{7,8}$. The observed cooling can be understood as being caused by the recent onset of neutron superfluidity in the core of the star, combined with a much earlier onset of proton superconductivity ${ }^{8,9}$. This has provided the first direct constraints on core superfluid and superconducting properties from neutron-star observations. These new results motivate studies of the possible implications. Here we explore the rotational evolution of young pulsars using the newly constrained superfluid properties and assuming this superfluid core is allowed to decouple (as discussed below). We use simulations ${ }^{10}$ of the cooling of a neutron star to determine the fraction of the neutron-star core that is superfluid as a function of time; this allows us to track the normal and superfluid components of the moment of inertia as the star ages (see Supplementary Fig. S1).

We consider a simple phenomenological model for the rotational evolution of the normal and superfluid components of the star:

$$
\begin{gathered}
\frac{\mathrm{d}}{\mathrm{d} t}(I \Omega)=-\beta \Omega^{3}-N_{\mathrm{pin}}-N_{\mathrm{mf}} \\
\frac{\mathrm{d}}{\mathrm{d} t}\left(I_{\mathrm{sf}} \Omega_{\mathrm{sf}}\right)=N_{\mathrm{pin}}+N_{\mathrm{mf}}
\end{gathered}
$$

where $I$ and $I_{\mathrm{sf}}$ are the moments of inertia of the normal and superfluid components, respectively, (and $I+I_{\mathrm{sf}}=$ constant) and $N_{\text {pin }}$ and $N_{\mathrm{mf}}$ are torques associated with vortex pinning ${ }^{11}$ and dissipative mutual friction ${ }^{12}$, respectively. Note that it is the rotation of the normal component $\Omega$ that is observed in pulsars. There are three simple limits that we can consider. The first is that friction acts on a much shorter timescale than the spin-down timescale ${ }^{12}$; this is the conventional view of rotational evolution, which leads to $\Omega_{\text {sf }}$ closely tracking $\Omega$ and a braking index $n=3$ (where $n \equiv \Omega \ddot{\Omega} / \dot{\Omega}^{2}$ and $\dot{x}$ and $\ddot{x}$ are the first and second, respectively, time derivatives of the parameter $x$ ), at odds with all measured values ${ }^{3}$. The second limit is when there is no pinning or friction; we find that this leads to $n>3$. The final case, which we consider in detail here, is when pinning causes $\dot{\Omega}_{\mathrm{sf}} \approx 0$. The evolution equations (1) and (2) can then be combined to give

$$
\frac{\mathrm{d} \Omega}{\mathrm{d} t}=\left(\Omega_{\mathrm{sf}}-\Omega\right) \frac{1}{I} \frac{\mathrm{d} I}{\mathrm{~d} t}-\beta \frac{\Omega^{3}}{I}
$$

The spin lag, $\Omega_{\mathrm{sf}}-\Omega$, is the difference in the rotational velocity between the superfluid and normal components. Some examples of decoupled spin evolution are shown in Fig. 1.

Conventional pulsar spin evolution accounts only for the second term on the right-hand side of equation (3), with constant $B$ and $I$. In this case, pulsars born at a particular spin period $(P=2 \pi / \Omega)$ evolve by moving along lines of constant ${ }^{13,14} B$, and the characteristic age $\tau_{\mathrm{c}}(\equiv P / 2 \dot{P})$ is an estimate of the true age of the pulsar. However, this again suggests that the conventional picture is incomplete: in cases where an independent age can be estimated (for example, from studying the expansion of an associated supernova remnant), the result is often quite different from the characteristic age; this can be seen in Table 1. When superfluid decoupling is taken into account, spin evolution is similar to the conventional course, except now the moment of 


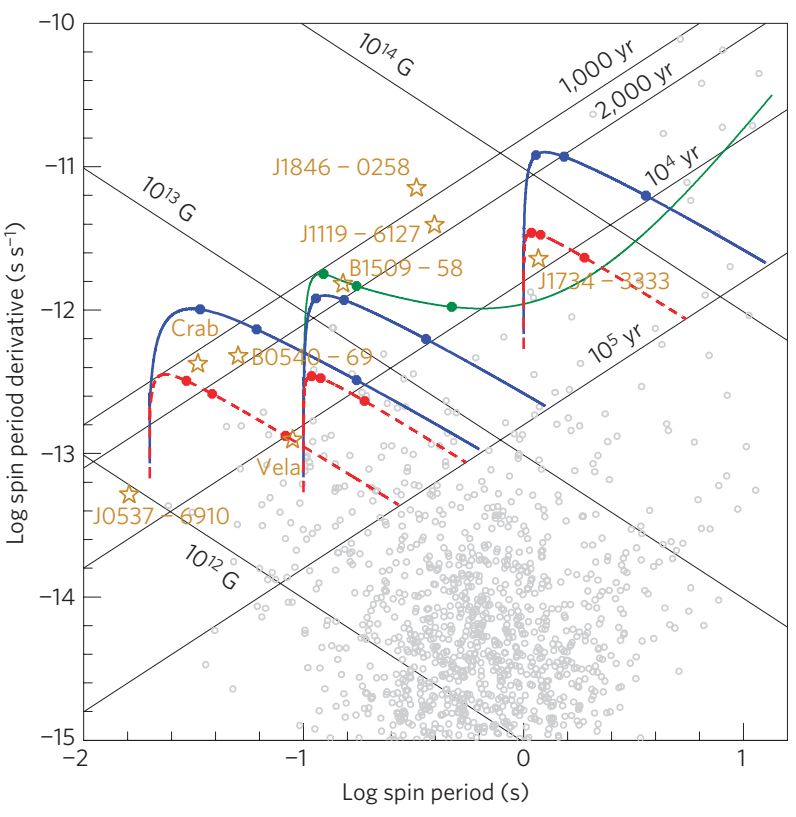

Figure 1 | Pulsar spin period versus spin period derivative. The open circles are observed values taken from the ATNF Pulsar Catalogue ${ }^{19}$. The stars denote pulsars with a measured braking index (see Table 1). The thin diagonal lines denote characteristic age $(=P / 2 \dot{P})$ and inferred magnetic field $\left(=3.2 \times 10^{19} \mathrm{G}(P \dot{P})^{1 / 2}\right)$. The curves are spin evolution tracks for pulsars with mass $1.8 \mathrm{M}_{\text {sun }}$ (red, dashed) and $1.4 \mathrm{M}_{\text {sun }}$ (blue, solid), where the spin lag is maintained at $\Omega_{\mathrm{sf}} / \Omega-1 \leq 10^{-6}$ (by an extra angular momentum sink); the filled circles denote the evolution at ages 1,000, 2,000 , and $10^{4} \mathrm{yr}$. Also shown (green, thin solid) is the evolution of a pulsar where the spin lag is allowed to grow. From left to right, the initial spin period and magnetic field $(P, B)$ are taken to be $\left(0.02 \mathrm{~s}, 5 \times 10^{12} \mathrm{G}\right),(0.1 \mathrm{~s}$, $\left.10^{13} \mathrm{G}\right)$ and $\left(1 \mathrm{~s}, 10^{14} \mathrm{G}\right)$. These examples demonstrate that the model can explain the observed pulsar population.

inertia decreases over time. If the spin lag remains small (for example, as a result of an angular momentum sink acting on the superfluid), only a small deviation (from evolution along a constant $B$ track) is seen at intermediate and late times (after $\sim 1,000-2,000 \mathrm{yr}$ ). Note that we are considering intermediate times $\left(\sim 10^{3}-10^{5} \mathrm{yr}\right)$ in the life of a neutron star, in between the short timescale for glitch recurrence ${ }^{15}(\sim 1 \mathrm{yr})$ and the long timescales for magnetic field diffusion ${ }^{13,14}$ and cooling ${ }^{16-18}\left(\sim 10^{5}-10^{6} \mathrm{yr}\right)$. On the other hand, if the spin lag is allowed to become large (for example, owing to strong vortex pinning), then we see that a decreasing $I$ can mimic a strongly increasing magnetic field, leading to pulsars evolving in the way suggested ${ }^{3}$ for PSR J1734-3333 with $n<3$. A further departure from the conventional picture is that the characteristic age is not an accurate indication of the true age of a pulsar at early times.

Not only are the spin period $P$ and first time derivative of the period $\dot{P}$ observable quantities, in some cases the second time derivative $\ddot{P}$ can be measured. This provides another test for our model. Table 1 gives data on eight systems where the braking index $n$, which is proportional to $\ddot{P}$, has been measured, whereas our model predicts the braking index to be (see equation (3))

$$
n=3-\frac{2 \dot{I}}{I} \frac{\Omega}{\dot{\Omega}}-\left(\frac{3 \dot{I}}{I} \frac{\Omega}{\dot{\Omega}}-\frac{\ddot{I}}{I} \frac{\Omega^{2}}{\dot{\Omega}^{2}}\right)\left(\frac{\Omega_{\mathrm{sf}}}{\Omega}-1\right)=3-4 \tau_{\mathrm{c}}\left|\frac{\dot{I}}{I}\right|
$$

where the second equality is obtained when $\Omega_{\mathrm{sf}}-\Omega \ll \Omega$.

As $\tau_{\mathrm{c}}$ and $n$ are observable quantities (related simply to $P, \dot{P}$ and $\ddot{P})$ for a given pulsar, we can compare our predictions to the pulsars in Table 1; this is shown in Fig. 2. For the Crab pulsar (the only one with a known age), we infer a relatively high mass of $\approx 1.8 M_{\text {Sun }}$ (for the particular equation of state and superfluid properties we consider; see Supplementary Information). Furthermore, we can use the mass determination to estimate the initial period and magnetic field of the pulsar, and we find an initial period $\sim 0.02 \mathrm{~s}$ and $B \sim 4 \times 10^{12} \mathrm{G}$.

The fact that our simple model is able to explain the observed pulsar properties demonstrates the merits of the notion of decoupled spin evolution due to the onset of core superfluidity. However, key questions remain to be answered. The main assumption in our model is that core superfluid neutrons are allowed to decouple and pin. Core pinning is thought to be the result of the interaction between superfluid vortices and flux tubes in the proton superconductor ${ }^{11}$. Whether this mechanism can be strong enough to act in the way assumed in our analysis is not clear at this time. Theoretical work is also required to determine whether the spin lag $\Omega_{\mathrm{sf}}-\Omega$ can be kept small during the evolution by an (at this time) unspecified angular momentum sink. From an observational point-of-view, discovery and long-term monitoring of a large number of systems by radio telescopes such as LOFAR and SKA will allow accurate timing of many young pulsars in the future. Taking these pulsars as an ensemble, we can constrain the nuclear equation of state and superfluid properties (because these determine the evolution of the moment of inertia), analogous to what is done in studies of neutron-star thermal evolution. Knowledge of these properties can then be used to infer the mass of individual pulsars. Finally, radio timing measurements may be

\section{Table 1 | Pulsars with observed braking index.}

\begin{tabular}{|c|c|c|c|c|c|c|}
\hline $\begin{array}{l}\text { Pulsar } \\
\text { name }\end{array}$ & $\begin{array}{l}\text { Supernova } \\
\text { remnant }\end{array}$ & $\begin{array}{l}\text { Period } \\
\text { (s) }\end{array}$ & $\begin{array}{l}\text { Period derivative } \\
\left(\mathrm{s} \mathrm{s}^{-1}\right)\end{array}$ & $\begin{array}{l}\text { Characteristic } \\
\text { age } \tau_{\mathrm{c}}(\mathrm{yr})\end{array}$ & $\begin{array}{l}\text { Braking } \\
\text { index } n\end{array}$ & $\begin{array}{l}\text { Age } \\
(y r)\end{array}$ \\
\hline $\mathrm{B} 0531+21$ & Crab & 0.0331 & $4.23 \times 10^{-13}$ & 1,240 & 2.51(1) (ref. 4) & 958 \\
\hline J0537 - 6910 & N157B & 0.0161 & $5.18 \times 10^{-14}$ & 4,930 & $-1.5(1)($ ref. 20) & $2,000_{-1,000}^{+3,000}($ ref. 21) \\
\hline B0540 - 69 & $0540-69.3$ & 0.0505 & $4.79 \times 10^{-13}$ & 1,670 & 2.140(9) (ref. 22) & $1,000_{-240}^{+660}($ ref. 23) \\
\hline B0833- 45 & Vela & 0.0893 & $1.25 \times 10^{-13}$ & 11,300 & $1.4(2)($ ref. 24) & $11,000_{-5,600}^{+5,000}($ ref. 25$)$ \\
\hline$J 1119-6127$ & $G 292.2-0.5$ & 0.408 & $4.02 \times 10^{-12}$ & 1,610 & $2.684(2)($ ref. 26) & $7,100_{-2,900}^{+500}($ ref. 27$)$ \\
\hline B1509- 58 & G320.4 - 1.2 & 0.151 & $1.54 \times 10^{-12}$ & 1,550 & $2.839(3)($ ref. 22) & $<21,000$ (ref. 28) \\
\hline $\mathrm{J} 1846-0258$ & Kesteven 75 & 0.325 & $7.08 \times 10^{-12}$ & 729 & $2.65(1)($ ref. 22) & $1,000_{-100}^{+3,300}$ (ref. 29) \\
\hline$J 1734-3333$ & G354.8- 0.8 & 1.17 & $2.28 \times 10^{-12}$ & 8,120 & $0.9(2)$ (ref. 3) & $>1,300$ \\
\hline
\end{tabular}

The periods and period derivatives are taken from ref. 19. The numbers in parentheses show the braking index uncertainty in the last digit. For J1734 - 3333, we give a lower limit of the age, which we estimate by considering the supernova remnant size (21 parsecs; ref. 30) and remnant expansion velocity $v_{S N R}$, to obtain an age $\sim 2,000 \mathrm{yr}\left(10^{4} \mathrm{~km} \mathrm{~s}{ }^{-1} / v_{\mathrm{SNR}}\right)$; and considering the pulsar's distance away from the centre of the supernova remnant (46 parsecs; ref. 30) and pulsar space velocity $v_{\text {pulsar, }}$ to obtain an age $\sim 23,000 \mathrm{yr}\left(2,000 \mathrm{~km} \mathrm{~s}^{-1} / \mathrm{v}_{\text {pulsar }}\right)$. 


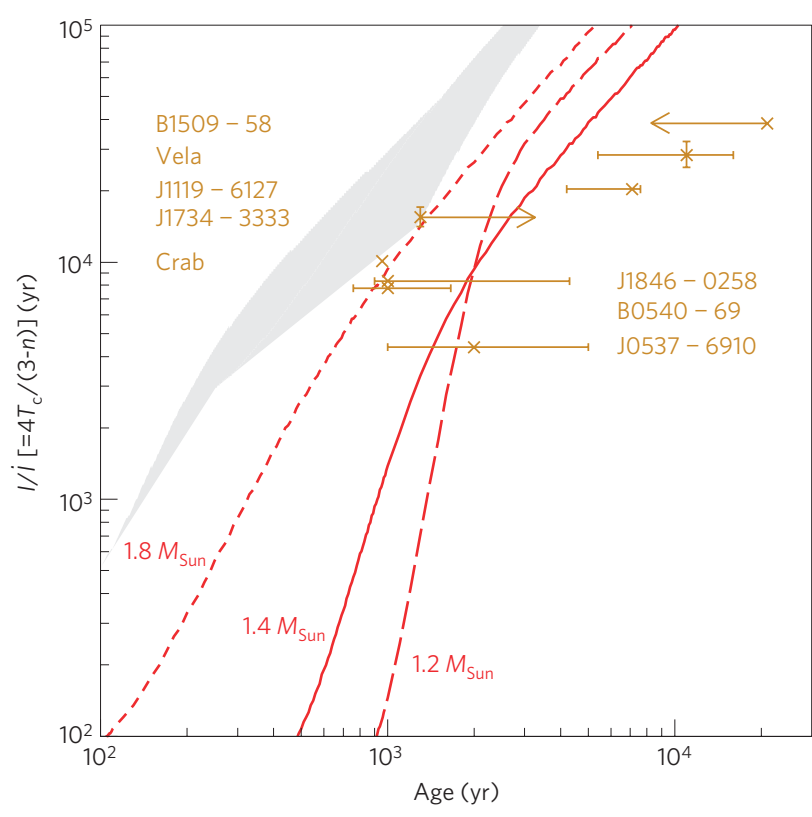

Figure 2 | Constraining neutron-star properties. The crosses denote pulsars with a measured braking index; horizontal bars and arrows indicate uncertainties or limits on their true age, and vertical bars are shown for pulsars with larger braking index uncertainty. The curves show the evolution of the effective moment of inertia for pulsars with mass $1.8 M_{\text {Sun }}$ (short-dashed), 1.4 M sun (solid), and 1.2 $M_{\text {sun }}$ (long-dashed), assuming a particular stellar and superfluid model ${ }^{9}$, and the shaded region represents an alternative model ${ }^{8}$; both sets of superfluid parameters fit the rapid cooling seen in the Cassiopeia A neutron star (see Supplementary Information) and provide examples of the effect of different $|I / i|$ models (see equation (4)). These moment-of-inertia evolution curves are analogous to the thermal cooling curves ${ }^{16-18}$ used to determine properties of neutron stars.

able to constrain neutron-star thermal evolution, independently of measurements at X-ray energies.

Note added in proof. After completion of this work, we became aware of the independent development of a similar mechanism for variation of the pulsar-braking index that was presented by $\mathrm{E}$. Kantor at two conferences in 2011 (http://go.nature.com/sxaWeN; http://go.nature.com/veAOt1).

Received 21 May 2012; accepted 15 August 2012; published online 30 September 2012

\section{References}

1. Pacini, F. Rotating neutron stars, pulsars and supernova remnants. Nature 219, 145-146 (1968).

2. Gunn, J. E. \& Ostriker, J. P. Magnetic dipole radiation from pulsars. Nature 221, 454-456 (1969).

3. Espinoza, C. M., Lyne, A. G., Kramer, M., Manchester, R. N. \& Kaspi, V. M. The braking index of PSR J1734-3333 and the magnetar population. Astrophys. J. 741, L13 (2011).

4. Lyne, A. G., Pritchard, R. S. \& Graham-Smith, F. 23 years of Crab pulsar rotational history. Mon. Not. R. Astron. Soc. 265, 1003-1012 (1993).

5. Migdal, A. B. Superfluidity and the moments of inertia of nuclei. Sov. Phys. JETP 10, 176-185 (1960).

6. Baym, G., Pethick, C. \& Pines, D. Superfluidity in neutron stars. Nature 224, 673-674 (1969).

7. Heinke, C. O. \& Ho, W. C. G. Direct observation of the cooling of the Cassiopeia A neutron star. Astrophys. J. 719, L167-L171 (2010).
8. Shternin, P. S., Yakovlev, D. G., Heinke, C. O., Ho, W. C. G. \& Patnaude, D. J. Cooling neutron star in the Cassiopeia A supernova remnant: Evidence for superfluidity in the core. Mon. Not. R. Astron. Soc. 412, L108-L112 (2011).

9. Page, D., Prakash, M., Lattimer, J. M. \& Steiner, A. W. Rapid cooling of the neutron star in Cassiopeia A triggered by neutron superfluidity in dense matter. Phys. Rev. Lett. 106, 081101 (2011).

10. Ho, W. C. G., Glampedakis, K. \& Andersson, N. Magnetars: Super(ficially) hot and super(fluid) cool. Mon. Not. R. Astron. Soc. 422, 2632-2641 (2012).

11. Link, B. Constraining hadronic superfluidity with neutron star precession. Phys. Rev. Lett. 91, 101101 (2003).

12. Alpar, M. A., Langer, S. A. \& Sauls, J. A. Rapid postglitch spin-up of the superfluid core in pulsars. Astrophys. J. 282, 533-541 (1984).

13. Goldreich, P. \& Reisenegger, A. Magnetic field decay in isolated neutron stars. Astrophys. J. 395, 250-258 (1992).

14. Glampedakis, K., Jones, D. I. \& Samuelsson, L. Ambipolar diffusion in superfluid neutron stars. Mon. Not. R. Astron. Soc. 413, 2021-2030 (2012).

15. Espinoza, C. M., Lyne, A. G., Stappers, B. W. \& Kramer, M. A study of 315 glitches in the rotation of 102 pulsars. Mon. Not. R. Astron. Soc. 414, 1679-1704 (2011).

16. Tsuruta, S. Thermal properties and detectability of neutron stars. II. Thermal evolution of rotation-powered neutron stars. Phys. Rep. 292, 1-130 (1998).

17. Yakovlev, D. G. \& Pethick, C. J. Neutron star cooling. Annu. Rev. Astron. Astrophys. 42, 169-210 (2004).

18. Page, D., Geppert, U. \& Weber, F. The cooling of compact stars. Nucl. Phys. A 777, 497-530 (2006).

19. Manchester, R. N., Hobbs, G. B., Teoh, A. \& Hobbs, M. The Australia Telescope National Facility Pulsar Catalogue. Astron. J. 129, 1993-2006 (2005).

20. Middleditch, J., Marshall, F. E., Wang, Q. D., Gotthelf, E. V. \& Zhang, W. Predicting the starquakes in PSR J0537-6910. Astrophys. J. 652, 1531-1546 (2006)

21. Chen, Y. et al. Chandra ACIS spectroscopy of N157B: A young composite supernova remnant in a superbubble. Astrophys. J. 651, 237-249 (2006).

22. Livingstone, M. A. et al. New phase-coherent measurements of pulsar braking indices. Astrophys. Space Sci. 308, 317-323 (2007).

23. Park, S., Hughes, J. P., Slane, P. O., Mori, K. \& Burrows, D. N. A deep Chandra observation of the oxygen-rich supernova remnant 0540-69.3 in the Large Magellanic Cloud. Astrophys. J. 710, 948-957 (2010).

24. Lyne, A. G., Pritchard, R. S., Graham-Smith, F. \& Camilo, F. Very low braking index for the Vela pulsar. Nature 381, 497-498 (1996).

25. Page, D., Lattimer, J. M., Prakash, M. \& Steiner, A. W. Neutrino emission from Cooper pairs and minimal cooling of neutron stars. Astrophys. J. 707, 1131-1140 (2009)

26. Weltevrede, P., Johnston, S. \& Espinoza, C. M. The glitch-induced identity changes of PSR J1119-6127. Mon. Not. R. Astron. Soc. 411, 1917-1934 (2011).

27. Kumar, H. S., Safi-Harb, S. \& Gonzalez, M. E. Chandra and XMM-Newton studies of the supernova remnant G292.2-0.5 associated with the pulsar J1119-6127. Astrophys. J. 754, 96 (2012).

28. Gaensler, B. M., Brazier, K. T. S., Manchester, R. N., Johnston, S. \& Green, A. J. SNR G320.4-01.2 and PSR B1509-58: New radio observations of a complex interacting system. Mon. Not. R. Astron. Soc. 305, 724-736 (1999).

29. Blanton, E. L. \& Helfand, D. J. ASCA observations of the composite supernova remnant G29.7-0.3. Astrophys. J. 470, 961-966 (1996).

30. Manchester, R. N. et al. in Neutron Stars in Supernova Remnants 31 (eds Slane, P. O. \& Gaensler, B. M.) (ASP Conf. Ser. 271, Astronomical Society of the Pacific, 2002).

\section{Acknowledgements}

W.C.G.H. thanks D. Yakovlev for providing equation-of-state tables. W.C.G.H. appreciates the use of the computer facilities at the Kavli Institute for Particle Astrophysics and Cosmology. W.C.G.H. and N.A. acknowledge support from the Science and Technology Facilities Council (STFC) in the United Kingdom.

\section{Author contributions}

W.C.G.H. contributed to developing the model, performed the calculations and wrote the manuscript. N.A. contributed to developing the model and writing the manuscript.

\section{Additional information}

Supplementary information is available in the online version of the paper. Reprints and permissions information is available online at www.nature.com/reprints.

Correspondence and requests for materials should be addressed to W.C.G.H. or N.A.

\section{Competing financial interests}

The authors declare no competing financial interests. 